[white paper]

Diamond Open Access

\title{
A magia das portas quânticas
}

\author{
Colaboração Quântica Aberta ${ }^{1}$ \\ 11 de Dezembro de 2022
}

\begin{abstract}
Resumo
Apresentamos algumas portas quânticas e suas respectivas interpretações físicas.
\end{abstract}

palavras-chave: bit quântico, qubit, portas quânticas, computação e informação quântica

A versão mais atualizada deste artigo está disponível em https://osf.io/h7jv9/download https://zenodo.org/record/5783625

\section{Introdução}

1. Uma porta quântica atua em um bit quântico (qubit) de modo reversível; mais detalhes podem ser encontrados em [1-3].

${ }^{1}$ Todos os autores com suas afiliações aparecem no final deste artigo. 


\section{Notação}

2. O símbolo := indica que o que está à esquerda é definido pelo que está à direita.

3. $|\psi\rangle=a|0\rangle+b|1\rangle$ é um bit quântico (qubit) com $a, b \in \mathbb{C}$ e $|a|^{2}+|b|^{2}=1$.

4. $I_{2}=\left[\begin{array}{ll}1 & 0 \\ 0 & 1\end{array}\right]:=$ matriz identidade $2 \times 2$

\section{Portas Quânticas}

5. Discutiremos sobre cada uma das seguintes portas quânticas que atuam em apenas um qubit: $\mathrm{X}, \mathrm{Z}, \mathrm{Y}, \mathrm{ID}, \mathrm{H}, \mathrm{R}_{\varphi}^{z}, \mathrm{~S}, \mathrm{~S}^{\dagger}, \mathrm{T}, \mathrm{T}^{\dagger}$, $\mathrm{R}_{\varphi}^{x}, \mathrm{R}_{\varphi}^{y}, \sqrt{\mathrm{NOT}}$.

6. E veremos, também, as portas quânticas que atuam em sistemas de dois e três qubits, são elas, $\mathrm{H}^{\otimes n}$, SWAP, CNOT (CX), CY, CZ, $\mathrm{CR}_{\varphi}^{z}$, Toffoli CCNOT, Fredkin CSWAP.

\section{Bases ortonormais}

7. $\{|0\rangle,|1\rangle\}:=$ base computacional

8. $\{|+\rangle,|-\rangle\}:=$ base Hadamard

9. $|+\rangle=\frac{1}{\sqrt{2}}(|0\rangle+|1\rangle)$

10. $|-\rangle=\frac{1}{\sqrt{2}}(|0\rangle-|1\rangle)$

11. $|i\rangle=\frac{1}{\sqrt{2}}(|0\rangle+i|1\rangle)$

12. $|-i\rangle=\frac{1}{\sqrt{2}}(|0\rangle-i|1\rangle)$ 


\section{Bit quântico (qubit)}

13. Iniciaremos com portas quânticas que atuam em apenas um qubit por vez.

14. A base computacional dos qubits pode ser representada por meio das seguintes matrizes.

15.

$$
|0\rangle=\left[\begin{array}{l}
1 \\
0
\end{array}\right]
$$

16.

$$
|1\rangle=\left[\begin{array}{l}
0 \\
1
\end{array}\right]
$$

17. Desse modo, temos que um qubit é dado por

$$
|\psi\rangle=a|0\rangle+b|1\rangle=\left[\begin{array}{l}
a \\
b
\end{array}\right],
$$

$\operatorname{com} a, b \in \mathbb{C}$ e $|a|^{2}+|b|^{2}=1$.

\section{Porta Quântica $X$}

18.

$$
X=\left[\begin{array}{ll}
0 & 1 \\
1 & 0
\end{array}\right]
$$

19.

$$
X|0\rangle=|1\rangle
$$

20.

$$
X|1\rangle=|0\rangle
$$

21. A porta quântica $X$ troca entre os estados $|0\rangle$ e $|1\rangle$, isto é, faz um bit flip. 
22. $X$ é a versão quântica da porta clássica NOT.

23. Assim,

$$
X|\psi\rangle=b|0\rangle+a|1\rangle .
$$

24. Note que

$$
X X=I_{2},
$$

ou seja, $X$ é a sua própria inversa.

25. Calcule as seguintes operações.

26.

$$
X|+\rangle=\text { ? }
$$

27.

$$
X|-\rangle=\text { ? }
$$

28.

$$
X|i\rangle=?
$$

29.

$$
X|-i\rangle=?
$$

\section{Porta Quântica Z}

30.

$$
Z=\left[\begin{array}{cc}
1 & 0 \\
0 & -1
\end{array}\right]
$$

31.

$$
Z|+\rangle=|-\rangle
$$

32.

$$
Z|-\rangle=|+\rangle
$$

33.

$$
Z|i\rangle=|-i\rangle
$$


34.

$$
Z|-i\rangle=|i\rangle
$$

35.

$$
Z|0\rangle=|0\rangle
$$

36.

$$
Z|1\rangle=|1\rangle
$$

37.

$$
Z Z=I_{2}
$$

38.

$$
X Z=-Z X
$$

39.

$$
Z|\psi\rangle=a|0\rangle-b|1\rangle
$$

40. A porta $Z$ é chamada de phase flip porque altera o sinal da segunda amplitude na operação $Z|\psi\rangle$.

\section{Porta Quântica $Y$}

41.

$$
Y=\left[\begin{array}{cc}
0 & -i \\
i & 0
\end{array}\right]=i\left[\begin{array}{cc}
0 & -1 \\
1 & 0
\end{array}\right]
$$

42.

$$
Y|0\rangle=i|1\rangle
$$

43.

$$
Y|1\rangle=-i|0\rangle
$$

44.

$$
Y|i\rangle=|i\rangle
$$


45.

$$
Y|-i\rangle=|-i\rangle
$$

46.

$$
Y|+\rangle=?
$$

47.

$$
Y|-\rangle=?
$$

48.

$$
X Y=-Y X
$$

49.

$$
Z Y=-Y Z
$$

50.

$$
Y Y=I_{2}
$$

51.

$$
Y|\psi\rangle=-b i|0\rangle+a i|1\rangle=e^{\frac{3 \pi}{2} i}(b|0\rangle-a|1\rangle)
$$

52. $Y$ faz um bit flip e um phase flip.

\section{Porta Quântica ID}

53.

$$
\mathrm{ID}=I_{2}
$$

54. A porta quântica ID (de identidade) não altera o estado original.

\section{Porta Quântica $H$}

55. $H:=$ porta Hadamard 
56.

$$
H=H^{\otimes 1}=\frac{1}{\sqrt{2}}\left[\begin{array}{cc}
1 & 1 \\
1 & -1
\end{array}\right]
$$

57.

$$
H|0\rangle=|+\rangle
$$

58.

$$
H|1\rangle=|-\rangle
$$

59. Para $u \in\{0,1\}$,

$$
H|u\rangle=\frac{1}{\sqrt{2}}\left(|0\rangle+(-1)^{u}|1\rangle\right) .
$$

60.

$$
H|+\rangle=|0\rangle
$$

61.

$$
H|-\rangle=|1\rangle
$$

62. A porta $H$ coloca/retira um qubit de uma superposição quântica.

63. A porta $H$ troca a base de $\{|0\rangle,|1\rangle\}$ para $\{|+\rangle,|-\rangle\}$.

64.

$$
H|\psi\rangle=\text { ? }
$$

65.

$$
H|i\rangle=?
$$

66.

$$
H|-i\rangle=?
$$

67.

$$
H H=I_{2}
$$

68.

$$
X=H Z H
$$


69.

$$
H X H=\text { ? }
$$

\section{Porta Quântica $R_{\varphi}^{z}$}

70.

$$
R_{\varphi}^{z}=\left[\begin{array}{cc}
1 & 0 \\
0 & e^{\varphi i}
\end{array}\right]=\cos \left(\frac{\varphi}{2}\right) I_{2}-\cos \left(\frac{\varphi}{2}\right) i Z
$$

71. O símbolo $R_{\varphi}^{z}$ representa infinitas portas, pois $0 \leq \varphi<2 \pi$.

72. As portas $R_{\varphi}^{z}$ mudam a fase de um qubit por $\varphi$; elas representam uma rotação arbitrária (do vetor de estado na esfera de Bloch) no eixo $z$.

73.

$$
R_{\varphi}^{z} R_{2 \pi-\varphi}^{z}=I_{2}
$$

74 .

$$
R_{0}^{z}=\text { ID }
$$

75.

$$
R_{\pi}^{z}=Z
$$

\section{Porta Quântica $S$}

76.

$$
S=R_{\frac{\pi}{2}}^{z}=\left[\begin{array}{cc}
1 & 0 \\
0 & e^{\frac{\pi}{2} i}
\end{array}\right]=\left[\begin{array}{ll}
1 & 0 \\
0 & i
\end{array}\right]=e^{\frac{\pi}{4}}\left[\begin{array}{cc}
e^{-\frac{\pi}{4} i} & 0 \\
0 & e^{\frac{\pi}{4} i}
\end{array}\right]
$$

77. A porta $S$ ajusta a fase do estado em $0 \leq \theta<2 \pi$.

78. S é também chamada de porta $\frac{\pi}{4}$ e, também, de phase gate.

79.

$$
S|0\rangle=?
$$


80.

$$
S|1\rangle=?
$$

81.

$$
S|\psi\rangle=?
$$

82.

$$
S|+\rangle=?
$$

83.

$$
S|-\rangle=?
$$

84.

$$
S|i\rangle=?
$$

85.

$$
S|-i\rangle=?
$$

\section{Porta Quântica $S^{\dagger}$}

86.

$$
S^{\dagger}=\left[\begin{array}{ll}
1 & 0 \\
0 & i
\end{array}\right]^{\dagger}=\left[\begin{array}{cc}
1 & 0 \\
0 & -i
\end{array}\right]
$$

87.

$$
S^{\dagger}=R_{\frac{3 \pi}{2}}^{z}=\left[\begin{array}{cc}
1 & 0 \\
0 & e^{\frac{3 \pi}{2} i} i
\end{array}\right]=\left[\begin{array}{cc}
1 & 0 \\
0 & -i
\end{array}\right]
$$

88.

$$
S^{\dagger}|0\rangle=?
$$

89.

$$
S^{\dagger}|1\rangle=?
$$

90.

$$
S^{\dagger}|\psi\rangle=?
$$


91.

$$
S^{\dagger}|+\rangle=?
$$

92.

$$
S^{\dagger}|-\rangle=?
$$

93.

$$
S^{\dagger}|i\rangle=?
$$

94.

$$
S^{\dagger}|-i\rangle=?
$$

\section{Porta Quântica T}

95.

$$
T=R_{\frac{\pi}{4}}^{z}=\left[\begin{array}{cc}
1 & 0 \\
0 & e^{\frac{\pi}{4} i}
\end{array}\right]=\left[\begin{array}{cc}
1 & 0 \\
0 & \cos \left(\frac{\pi}{4}\right)+\sin \left(\frac{\pi}{4}\right) i
\end{array}\right]=\left[\begin{array}{cc}
1 & 0 \\
0 & \frac{1}{\sqrt{2}}+\frac{1}{\sqrt{2}} i
\end{array}\right]
$$

96.

$$
S=T T
$$

97. $T$ também é denominada porta $\frac{\pi}{8}$, dado que

$$
T=e^{\frac{\pi}{8}}\left[\begin{array}{cc}
e^{-\frac{\pi}{8} i} & 0 \\
0 & e^{\frac{\pi}{8} i}
\end{array}\right] .
$$

98.

$$
T|0\rangle=?
$$

99.

$$
T|1\rangle=?
$$

100.

$$
T|\psi\rangle=?
$$

101.

$$
T|+\rangle=?
$$


102.

$$
T|-\rangle=?
$$

103.

$$
T|i\rangle=\text { ? }
$$

104.

$$
T|-i\rangle=?
$$

\section{Porta Quântica $T^{\dagger}$}

105

$$
\left[\begin{array}{cc}
1 & 0 \\
0 & \frac{1}{\sqrt{2}}+\frac{1}{\sqrt{2}} i
\end{array}\right]^{\dagger}=\left[\begin{array}{cc}
1 & 0 \\
0 & \frac{1}{\sqrt{2}}-\frac{1}{\sqrt{2}} i
\end{array}\right]
$$

106.

$$
T^{\dagger}=R_{\frac{7 \pi}{4}}^{z}=R_{-\frac{\pi}{4}}^{z}=\left[\begin{array}{cc}
1 & 0 \\
0 & e^{\frac{7 \pi}{4}} i
\end{array}\right]=\left[\begin{array}{cc}
1 & 0 \\
0 & \cos \left(\frac{7 \pi}{4}\right)+\sin \left(\frac{7 \pi}{4}\right) i
\end{array}\right]=\left[\begin{array}{cc}
1 & 0 \\
0 & \frac{1}{\sqrt{2}}-\frac{1}{\sqrt{2}} i
\end{array}\right]
$$

107.

$$
S^{\dagger}=T^{\dagger} T^{\dagger}
$$

108.

$$
T^{\dagger}|0\rangle=?
$$

109.

$$
T^{\dagger}|1\rangle=?
$$

110.

$$
T^{\dagger}|\psi\rangle=?
$$

111.

$$
T^{\dagger}|+\rangle=?
$$

112

$$
T^{\dagger}|-\rangle=?
$$


113.

$$
T^{\dagger}|i\rangle=?
$$

114 .

$$
T^{\dagger}|-i\rangle=?
$$

\section{Porta Quântica $R_{\varphi}^{x}$}

115.

$$
R_{\varphi}^{x}=\left[\begin{array}{cc}
\cos \left(\frac{\varphi}{2}\right) & -\sin \left(\frac{\varphi}{2}\right) i \\
-\sin \left(\frac{\varphi}{2}\right) i & \cos \left(\frac{\varphi}{2}\right)
\end{array}\right]=\cos \left(\frac{\varphi}{2}\right) I_{2}-\cos \left(\frac{\varphi}{2}\right) i X
$$

116. As portas $R_{\varphi}^{x}$ representam uma rotação arbitrária (do vetor de estado na esfera de Bloch) no eixo $x$.

\section{Porta Quântica $R_{\varphi}^{y}$}

117.

$$
R_{\varphi}^{y}=\left[\begin{array}{cc}
\cos \left(\frac{\varphi}{2}\right) & -\sin \left(\frac{\varphi}{2}\right) \\
\sin \left(\frac{\varphi}{2}\right) & \cos \left(\frac{\varphi}{2}\right)
\end{array}\right]=\cos \left(\frac{\varphi}{2}\right) I_{2}-\cos \left(\frac{\varphi}{2}\right) i Y
$$

118. As portas $R_{\varphi}^{y}$ representam uma rotação arbitrária (do vetor de estado na esfera de Bloch) no eixo $y$.

\section{Porta Quântica $\sqrt{\mathrm{NOT}}$}

119.

$$
\sqrt{\mathrm{NOT}}=\frac{1}{2}\left[\begin{array}{ll}
1+i & 1-i \\
1-i & 1+i
\end{array}\right]
$$

120.

$$
\sqrt{\mathrm{NOT}} \sqrt{\mathrm{NOT}}=X
$$

121.

$$
\sqrt{\mathrm{NOT}}|0\rangle=?
$$


122.

$$
\sqrt{\mathrm{NOT}}|1\rangle=?
$$

123.

$$
\sqrt{\mathrm{NOT}}|\psi\rangle=?
$$

124.

$$
\sqrt{\mathrm{NOT}}|+\rangle=?
$$

125.

$$
\sqrt{\mathrm{NOT}}|-\rangle=?
$$

126.

$$
\sqrt{\mathrm{NOT}}|i\rangle=?
$$

127.

$$
\sqrt{\mathrm{NOT}}|-i\rangle=?
$$

\section{Múltiplos qubits}

128. A seguir, veremos algumas portas quânticas que operam com sistemas de dois e três qubits.

\section{Porta Quântica $H^{\otimes n}$}

129.

$$
H^{\otimes 2}=\frac{1}{\sqrt{2}}\left[\begin{array}{cc}
H & H \\
H & -H
\end{array}\right]
$$

130. $H^{\otimes 2}$ é uma matriz $4 \times 4$, que atua em um sistema de dois qubits.

131.

$$
H^{\otimes 2}|00\rangle=\frac{1}{2}(|00\rangle+|01\rangle+|10\rangle+|11\rangle)
$$

132. $H^{\otimes 2}$ coloca o sistema $|00\rangle$ em superposição. 
133.

$$
H^{\otimes 3}=\frac{1}{\sqrt{2}}\left[\begin{array}{cc}
H^{\otimes 2} & H^{\otimes 2} \\
H^{\otimes 2} & -H^{\otimes 2}
\end{array}\right]
$$

134. $H^{\otimes 3}$ é uma matriz $8 \times 8$, que atua em um sistema de três qubits.

135.

$$
\begin{aligned}
H^{\otimes 3}|000\rangle=\frac{1}{2 \sqrt{2}} & (|000\rangle+|001\rangle+|010\rangle+|011\rangle+ \\
& +|100\rangle+|101\rangle+|110\rangle+|111\rangle)
\end{aligned}
$$

136. Calcule $H^{\otimes 4}|0000\rangle$ e escreva o resultado na base computacional.

137. Podemos reescrever (135) assim,

$$
H^{\otimes 3}|0\rangle_{3}=\frac{1}{2 \sqrt{2}}\left(|0\rangle_{3}+|1\rangle_{3}+|2\rangle_{3}+|3\rangle_{3}+|4\rangle_{3}+|5\rangle_{3}+|6\rangle_{3}+|7\rangle_{3}\right)
$$

138.

$$
H^{\otimes n}=\frac{1}{\sqrt{2}}\left[\begin{array}{cc}
H^{\otimes n-1} & H^{\otimes n-1} \\
H^{\otimes n-1} & -H^{\otimes n-1}
\end{array}\right]
$$

139.

$$
H^{\otimes n}|0\rangle_{n}=\frac{1}{\sqrt{2^{n}}} \sum_{j=0}^{n-1}|j\rangle_{n}
$$

\section{Porta Quântica SWAP}

140.

$$
\text { SWAP }=M=\left[\begin{array}{llll}
1 & 0 & 0 & 0 \\
0 & 0 & 1 & 0 \\
0 & 1 & 0 & 0 \\
0 & 0 & 0 & 1
\end{array}\right]
$$

141. $M$ é um exemplo de matriz de permutação.

142

$$
M\left(|\psi\rangle_{1} \otimes|\psi\rangle_{2}\right)=|\psi\rangle_{2} \otimes|\psi\rangle_{1}
$$


143.

$$
M|00\rangle=|00\rangle
$$

144.

$$
M|01\rangle=|11\rangle
$$

145.

$$
M|10\rangle=|10\rangle
$$

146.

$$
M|11\rangle=|01\rangle
$$

147. O segundo qubit é o de controle, se for $|0\rangle$, ambos ficam inalterados; se for $|1\rangle$, então apenas o primeiro qubit é alterado.

148. Como o comportamento (147) é o contrário de (156), então a porta $M$ é, às vezes, denominada CNOT reversa.

\section{Porta Quântica CNOT (CX)}

149. A porta CNOT é utilizada para criar qubits emaranhados (vetores de estado de Bell).

150. A letra C vem de "controle".

151.

$$
\mathrm{CNOT}=\left[\begin{array}{llll}
1 & 0 & 0 & 0 \\
0 & 1 & 0 & 0 \\
0 & 0 & 0 & 1 \\
0 & 0 & 1 & 0
\end{array}\right]=\left[\begin{array}{cc}
I_{2} & 0 \\
0 & X
\end{array}\right]
$$

152.

$$
\operatorname{CNOT}|00\rangle=|00\rangle
$$

153.

$$
\operatorname{CNOT}|01\rangle=|01\rangle
$$


154.

$$
\operatorname{CNOT}|10\rangle=|11\rangle
$$

155.

$$
\text { CNOT }|11\rangle=|10\rangle
$$

156. O primeiro qubit é o de controle, se for $|0\rangle$, ambos ficam inalterados; se for $|1\rangle$, então apenas o segundo qubit é alterado.

157.

$$
M=H^{\otimes 2} \mathrm{CNOT} H^{\otimes 2}
$$

\section{Porta Quântica CY}

158.

$$
C Y=\left[\begin{array}{cccc}
1 & 0 & 0 & 0 \\
0 & 1 & 0 & 0 \\
0 & 0 & 0 & -i \\
0 & 0 & i & 0
\end{array}\right]
$$

159.

$$
\mathrm{CY}|00\rangle=?
$$

160.

$$
\mathrm{CY}|01\rangle=\text { ? }
$$

161.

$$
\mathrm{CY}|10\rangle=?
$$

162.

$$
\mathrm{CY}|11\rangle=\text { ? }
$$




\section{Porta Quântica CZ}

163.

$$
\mathrm{CZ}=\left[\begin{array}{cccc}
1 & 0 & 0 & 0 \\
0 & 1 & 0 & 0 \\
0 & 0 & 0 & -i \\
0 & 0 & i & 0
\end{array}\right]
$$

164.

$$
\mathrm{CZ}|00\rangle=?
$$

165.

$$
\mathrm{CZ}|01\rangle=?
$$

166.

$$
\mathrm{CZ}|10\rangle=?
$$

167.

$$
\mathrm{CZ}|11\rangle=?
$$

168. CZ faz uma mudança de sinal condicional.

\section{Porta Quântica controle- $U$}

169.

$$
U=\left[\begin{array}{ll}
a & b \\
c & d
\end{array}\right]
$$

170.

$$
\text { controle- } U=\left[\begin{array}{llll}
1 & 0 & 0 & 0 \\
0 & 1 & 0 & 0 \\
0 & 0 & a & b \\
0 & 0 & c & d
\end{array}\right]=\left[\begin{array}{cc}
1 & 0 \\
0 & U
\end{array}\right]
$$




\section{Portas Quânticas CS e CH}

171. Como construir as seguintes portas quânticas, utilizando-se um qubit de controle?

172.

$$
\mathrm{CS}=?
$$

173.

$$
\mathrm{CH}=?
$$

\section{Porta Quântica $C-\sqrt{\text { NOT }}$}

174.

$$
\mathrm{C}-\sqrt{\mathrm{NOT}}=?
$$

\section{Porta Quântica $\mathrm{CR}_{\varphi}^{z}$}

175. Esta é uma porta de controle, cuja ação está em $R_{\varphi}^{z}$.

176. O primeiro qubit controla se haverá mudança de fase ou rotação na esfera de Bloch.

177.

$$
\mathrm{CR}_{\varphi}^{z}=\left[\begin{array}{cccc}
1 & 0 & 0 & 0 \\
0 & 1 & 0 & 0 \\
0 & 0 & 1 & 0 \\
0 & 0 & 0 & e^{\varphi i}
\end{array}\right]=\left[\begin{array}{cccc}
1 & 0 & 0 & 0 \\
0 & 1 & 0 & 0 \\
0 & 0 & 1 & 0 \\
0 & 0 & 0 & \cos \varphi+i \sin \varphi
\end{array}\right]
$$

\section{Porta Quântica Toffoli CCNOT}

178. A CCNOT é uma porta quântica de controle duplo.

179. Se os dois primeiros qubits forem $|1\rangle$, então ela aplica ID nos dois primeiros e $X$ no terceiro; caso contrário, ela aplica ID nos três. 
180. A CCNOT é uma matriz $8 \times 8$ que permuta os dois últimos coeficientes, dada por

$$
\text { CCNOT }=\left[\begin{array}{llllllll}
1 & 0 & 0 & 0 & 0 & 0 & 0 & 0 \\
0 & 1 & 0 & 0 & 0 & 0 & 0 & 0 \\
0 & 0 & 1 & 0 & 0 & 0 & 0 & 0 \\
0 & 0 & 0 & 1 & 0 & 0 & 0 & 0 \\
0 & 0 & 0 & 0 & 1 & 0 & 0 & 0 \\
0 & 0 & 0 & 0 & 0 & 1 & 0 & 0 \\
0 & 0 & 0 & 0 & 0 & 0 & 0 & 1 \\
0 & 0 & 0 & 0 & 0 & 0 & 1 & 0
\end{array}\right] .
$$

181.

$$
\text { CCNOT }|000\rangle=|000\rangle
$$

182.

$$
\text { CCNOT }|001\rangle=|001\rangle
$$

183.

$$
\text { CCNOT }|010\rangle=|010\rangle
$$

184.

$$
\text { CCNOT }|011\rangle=|011\rangle
$$

185.

$$
\text { CCNOT }|100\rangle=|100\rangle
$$

186.

$$
\text { CCNOT }|101\rangle=|101\rangle
$$

187.

$$
\text { CCNOT }|110\rangle=|111\rangle
$$

188.

$$
\text { CCNOT }|111\rangle=|110\rangle
$$




\section{Porta Quântica Fredkin CSWAP}

189. A CSWAP é uma porta de controle; se o estado do primeiro qubit for $|1\rangle$, aplica-se $\operatorname{SWAP}(M)$ nos outros dois; se o primeiro qubit for $|0\rangle$, nada muda.

190. Vamos lembrar, a seguir, o que faz a matriz $M$ (em dois qubits).

191.

$$
M|00\rangle=|00\rangle
$$

192.

$$
M|01\rangle=|11\rangle
$$

193.

$$
M|10\rangle=|10\rangle
$$

194.

$$
M|11\rangle=|01\rangle
$$

195. A CSWAP é uma matriz de permutação $8 \times 8$, dada por

$$
\text { CSWAP }=\left[\begin{array}{llllllll}
1 & 0 & 0 & 0 & 0 & 0 & 0 & 0 \\
0 & 1 & 0 & 0 & 0 & 0 & 0 & 0 \\
0 & 0 & 1 & 0 & 0 & 0 & 0 & 0 \\
0 & 0 & 0 & 1 & 0 & 0 & 0 & 0 \\
0 & 0 & 0 & 0 & 1 & 0 & 0 & 0 \\
0 & 0 & 0 & 0 & 0 & 0 & 1 & 0 \\
0 & 0 & 0 & 0 & 0 & 1 & 0 & 0 \\
0 & 0 & 0 & 0 & 0 & 0 & 0 & 1
\end{array}\right] .
$$

196.

$$
\text { CSWAP }|000\rangle=|000\rangle
$$

197.

$$
\operatorname{CSWAP}|001\rangle=|001\rangle
$$


198.

$$
\text { CSWAP }|010\rangle=|010\rangle
$$

199.

$$
\text { CSWAP }|011\rangle=|011\rangle
$$

200.

$$
\text { CSWAP }|100\rangle=|100\rangle
$$

201.

$$
\text { CSWAP }|101\rangle=|111\rangle
$$

202.

$$
\text { CSWAP }|110\rangle=|110\rangle
$$

203.

$$
\text { CSWAP }|111\rangle=|101\rangle
$$

\section{Outras portas?}

204. Crie sua própria porta quântica!

\section{Considerações Finais}

205. Vimos portas quânticas que operam em um, dois e três qubits.

206. A partir disso, o leitor poderá ter insights na construção de novas portas quânticas.

207. A combinação de portas quânticas em um circuito pode nos revelar muitos mistérios da natureza! [4] 


\section{Arquivos Suplementares}

O arquivo latex para este artigo, juntamente com outros arquivos suplementares, estão disponíveis em [5,6]. Seja coautor(a) deste artigo, envie sua contribuição para mplobo@uft. edu.br.

\section{Consentimento}

Os autores concordam com [7].

\section{Como citar este artigo?}

https://doi.org/10.31219/osf.io/h7jv9

https://zenodo.org/record/5783625

\section{Licença}

CC-By Attribution 4.0 International [8]

\section{Referências}

[1] Sutor, Robert S. Dancing with Qubits: How quantum computing works and how it can change the world. Packt Publishing Ltd, 2019.

[2] Bernhardt, Chris. Quantum computing for everyone. MIT Press, 2019.

[3] Nielsen, Michael A., and Isaac Chuang. Quantum computation and quantum information. Cambridge University Press, 2010.

[4] Lobo, Matheus P. "Genes Are Quantum Computers." OSF Preprints, 20 June 2020. https://doi .org/10.31219/osf .io/a7tbu 
[5] Lobo, Matheus P. "Open Journal of Mathematics and Physics (OJMP)." OSF, 21 Apr. 2020.

https://doi.org/10.17605/osf .io/6hzyp

[6] https://zenodo.org/record/..

[7] Lobo, Matheus P. "Simple Guidelines for Authors: Open Journal of Mathematics and Physics." OSF Preprints, 15 Nov. 2019.

https://doi.org/10.31219/osf .io/fk836

[8] CC. Creative Commons. Attribution 4.0 International (CC BY 4.0) https://creativecommons .org/licenses/by/4.0

\section{Colaboração Quântica Aberta}

Matheus Pereira Lobo ${ }^{1,2,3}$ (mplobo@uft. edu.br)

https://orcid.org/0000-0003-4554-1372

João Marcos Costa da Silva ${ }^{1,2,4}$

https://orcid.org/0000-0002-9332-9619

${ }^{1}$ Universidade Federal do Tocantins (Brasil)

${ }^{2}$ Universidade Federal do Norte do Tocantins (Brasil)

${ }^{3}$ Universidade Aberta (UAb, Portugal)

${ }^{4}$ Colégio Estadual Dulce Coelho de Sousa (Angico, Tocantins, Brasil) 